\title{
Formal Modeling and Analysis for Power Grid Operation Risk
}

\author{
Lin Cheng, Rui Chen, Mingshun Liu, Tanfeng Ma and Zhonghai Ma \\ Guizhou Power Grid Co.,Ltd, Guiyang, China \\ E-Link Information Technique Co.,Ltd, Shenzhen, China

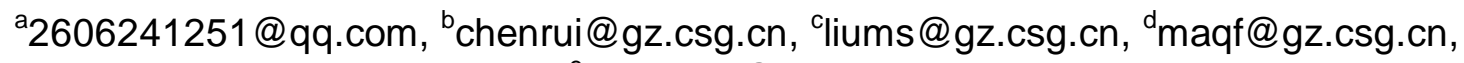 \\ 55454604@qq.com
}

Keywords: Risk analysis; Formal modeling; FMEA; Electric system

\begin{abstract}
As more and more coupled facilities being integrated into the electric system, the system operation is changing into a high risk level. The spreading of a small failure can result a big disaster in many large-scale electric systems. Failure modes and effects analysis (FMEA) is a conventional risk analysis approach which has a worldwide recognition in the past years. This paper proposes a formal modeling approach for electric system operation risk, in which the failure modes are defined as the inherent features of the system components. Then, we propose a structured modeling technique for representing FMEA causal knowledge in unified Boolean matrices. By searching the reasoning matrices iteratively, the system effects of the certain bottom-level failure events can be found automatically. The result points out more important focus to handle, advance the treatment order of the failure events, and so improve the efficiency of risk management.
\end{abstract}

\section{Introduction}

As the large-scale electric systems experiencing a more complicated trend, the risk probability is also experiencing a higher trend, especially when considering the environment and human factors [1]. FMEA is recognized as an important risk analysis method to help determine the risk value and treatment strategies [2]. By analyzing the potential failure modes and the effects on the components of the underlying system, the failure modes are classified according to their hazardous degree. Moreover, the preventive and ameliorative measures are provided to improve the safety and reliability of the complex system. However, the conventional FMEA techniques are insufficient in aspect of the semantics and logical architecture to provide formal knowledge representation and traceable mechanism [3]. As a result, the realization of automated FMEA becomes very difficult in practice.

For that reason, this paper proposes a new modeling technique to represent failure causal knowledge formally. At first, it establishes the mapping relationship between the sheet FMEA and the entities in different system hierarchy. Then, it provides an advanced framework to organize the available causal knowledge about the underlying system. In addition, the Boolean matrices as a standardized mathematical model are utilized to store the FMEA causal knowledge. So that the causal knowledge and structure components can be represent in consistent mathematic language. Owing to the advantageous characteristic of the formal knowledge representation, it can be helpful for the subsequent causal reasoning and effect analysis. On the other hand, applying the computer technique to FMEA can improve the efficient and quality of the analysis process.

\section{FMEA Overview}

FMEA is a conventional deductive risk analysis technique which is applied during the system design and usage stage. Generally, the methodology includes four steps as follows.

- Knowledge Model: employing the object-oriented technique to represent the expert's experiences and knowledge with typical unit model.

- System Modeling: system model is abstract description of structure of the system, also the base of risk analysis. 
- Failure Mode Selection: confirming all the conceivable failure modes under specific system operational condition.

- Failure Effect Reasoning: deducing effect of the selected failure modes based on the system model.

Traditional FMEA relies on the analyzer's knowledge and skill greatly, and it is subjective process in some sense. As a consequence, the analysis result is often not satisfied for the large-scale complicated systems. Many researchers studied on the computer-aided FMEA in the past years. However, these techniques often suffer from a lack of formal semantics to represent the correctness or consistency of the descriptions. Considering the power grid is often a large-scale electric system, we need a computer-aided method to improve the efficiency of FMEA.

\section{Formal Modeling for FMEA}

Hierarchical Structure Decomposition. In general, it is difficult and needless to analyze the whole system synchronously. To obtain a reasonable range of analysis, we can decompose the large electric system into a hierarchical architecture to help the analysis. In the hierarchical structure model, the entire system is constituted with several subsystems which are made of many sub-sub-systems, and so on. In this way, we can decompose the complex electric system iteratively into the smallest replaceable unit, such as a transformer substation, voltage changer or ammeter [4,5]. The delimitation of system hierarchy is optional and the decomposition process is subjective. Each system level is constituted of one or more components and the granularity of the system decomposition increases while taking the more low level into account. Constructing the system hierarchical structure models is an intuitive process, but it is useful for understanding the system's infrastructure.

Failure Causal Relationship. Through the system decomposition, we can obtain the distinct components in every level under the granularity defined in advance. Analogously, we can track the original failure causes of each failure mode until the bottom level is arrived. Considering the failure maybe happens in either level, the phenomena and mechanism would be complicated because of the interdependence and interaction among the failure modes and their carriers. A failure mode perhaps occurs due to more than one component's breakdown, and one component's failure may be caused by more than one failure mode. To represent the potential relationship between the components and their failure modes, we provide an integrated mapping framework in which the directional lines indicate the corrective relationships, as shown in Fig. 1. Through the mapping framework, the failure modes are related to the corresponding components by regarding the failure modes as inherent characteristics of the components.

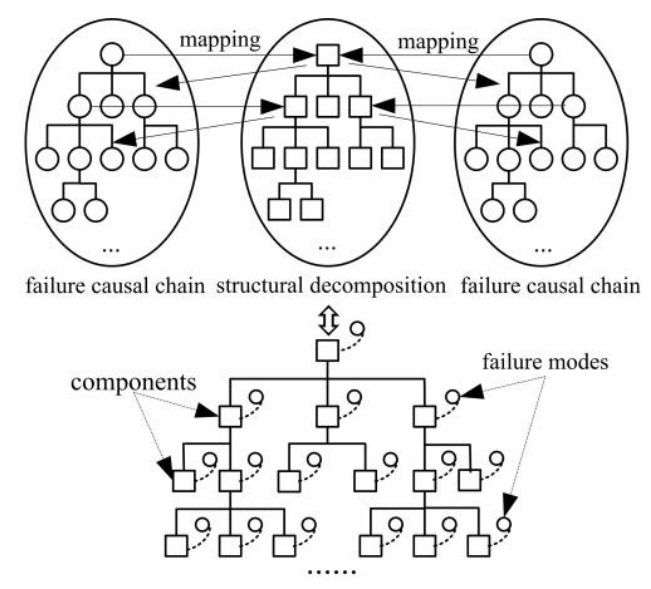

Fig.1 Mapping framework

The failure of large- scale electric system are complicated due to the interdependence among the failure modes. The traditional approach to risk analysis is to find all the failure modes, their causes and their effects. From the mapping framework above, we can find that the failure modes in different hierarchies have an intrinsic causal relationship. For each failure mode occurring in the certain level, its 
direct effects are usually the failure modes in the next higher level, and its direct causes are the failure modes in the next lower level. Therefore, we can trace the final effects and original causes along the structure decomposition framework until the top and the bottom level is arrived, for example, by turns the system, the subsystems, the subassemblies and parts etc. Generally, the bottom level components' failures can be considered as the source of the system failure, and the failure modes of these components can be defined as the original causes. When the bottom level components fail in a certain amount, the entire system will breakdown immediately or increase the deterioration step-by-step. In the real complex electric system, there are other factors need to be considered such as human, climate and environment etc., which need to be merged into the model for facilitating the risk analysis.

Formal FMEA Knowledge Representation Model. Traditional spreadsheet-based is short of sharing and reusing the failure knowledge because of its unstructured knowledge representation. Considering the potential structural relationship between the components and the failure modes, we can assign each failure mode to a corresponding component and define it as an inherent property of the component. To facilitate the realization in computer, the Boolean matrix can be utilized to store the causal relationships. We can use a element set $A=\left(a_{1}, \ldots, a_{i}, \ldots, a_{n}\right)$ to express a set of components at one level, then the element property $F_{j}\left(a_{i}\right)$ can be assigned to represent the single failure mode of the component $a_{i}$, while the element property set $F\left(a_{i}\right)$ corresponding to the whole. In this way, we can construct a formal FMEA knowledge representation model for the complex system as shown in Fig. 2.

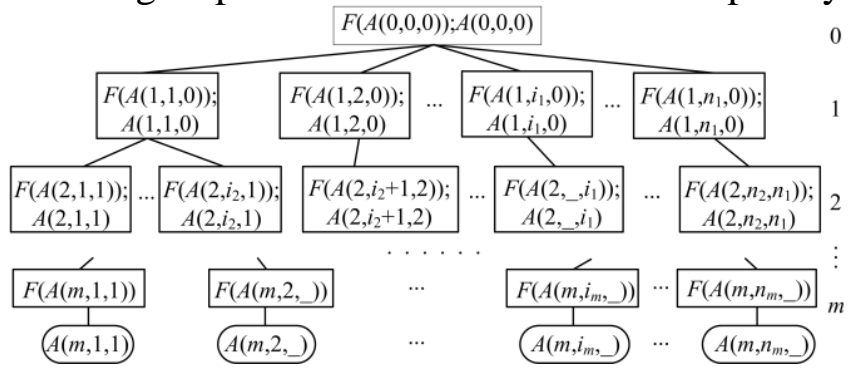

Fig. 2 Formal FMEA knowledge representation model

In the formal model, the component and its failure modes are assembled and expressed as a 2-tuple $<F\left(A\left(k, i_{k}, j_{k-1}\right)\right) ; A\left(k, i_{k}, j_{k-1}\right)>$ where $A\left(k, i_{k}, j_{k-1}\right)$ denotes the $i_{k}$ th component at the $k$ th level and $F\left(A\left(k, i_{k}\right.\right.$, $\left.j_{k-1}\right)$ ) indicates a set of failure modes of the $i_{k}$ th component. Additionally, $A\left(k, i_{k}, j_{k-1}\right)$ is also the subcomponent of the $j_{k-1}$ th component at the $(k-1)$ th level. Particularly, the 2-tuple $<F(A(0,0,0)) ; A(0,0,0)\rangle$ is the top node in which $A(0,0,0)$ represents the whole system and $F(A(0,0,0))$ represents the corresponding top-level failure modes. $n_{k}$ is the total number of nodes at the $k$ th level.

As has been noted, the failure modes in the bottom level can be regarded as the original causes of system fault. These failure modes should be prepared with more detail descriptions in advance, and can be expressed within the 2-tuple set $\left(S_{b}\right)$

$$
S_{b}=\left\{\left\langle a_{i}, F\left(a_{i}\right)\right\rangle \mid i=1,2, \cdots, n\right\}
$$

where $a_{i}$ is the bottom level component and $F\left(a_{i}\right)$ is a set of corresponding failure modes. The knowledge accumulation of the original FMEA is depended on the experience of the analyzers and it is very important for the electric system operation risk analysis.

As previously outlined, the Boolean matrix can be employed as an available instrument to store the causal relationships existing between the components and their failure modes. To gain the possible failure effects or locate the specific fault positions of the system, we can search the reasoning matrices iteratively. The reasoning matrix can be expressed as follows: 


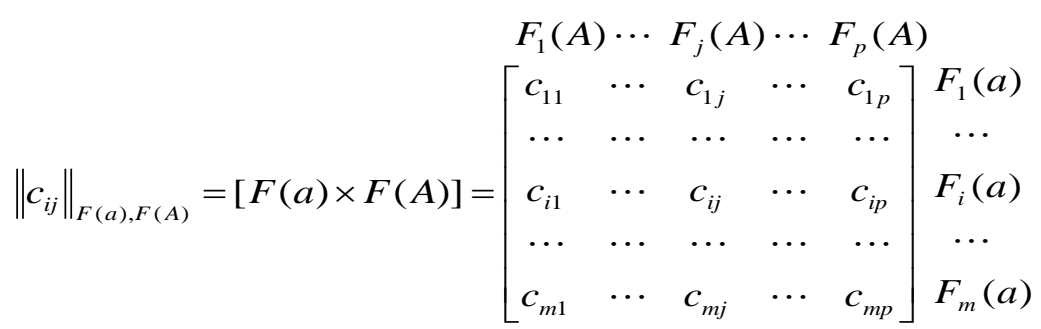

where $c_{i j}=1$ denotes that there is a causal relationship between the single failure mode $F_{i}(a)$ and the failure mode $F_{j}(A)$ in the next high level, and $c_{i j}=0$, for the opposite case.

\section{Risk Analysis Process}

System risk analysis should find each possible failure modes in low level and their effects on the global system. The automated FMEA begins with the components in the selected level, and all the possible failure modes should be identified in advance. Through the Boolean matrices we can assesses the effect on any system level, both the local and the next higher level. However, the failure effects on different system levels are not acquirable directly. In general, the local level and the next upper level will be influenced at first, and the entire system will be disabled or fall into breakdown state while the end effect is got through upwards circularly. The risk analysis process is described in Fig. 3 which reveals the specific procedure for automated FMEA.

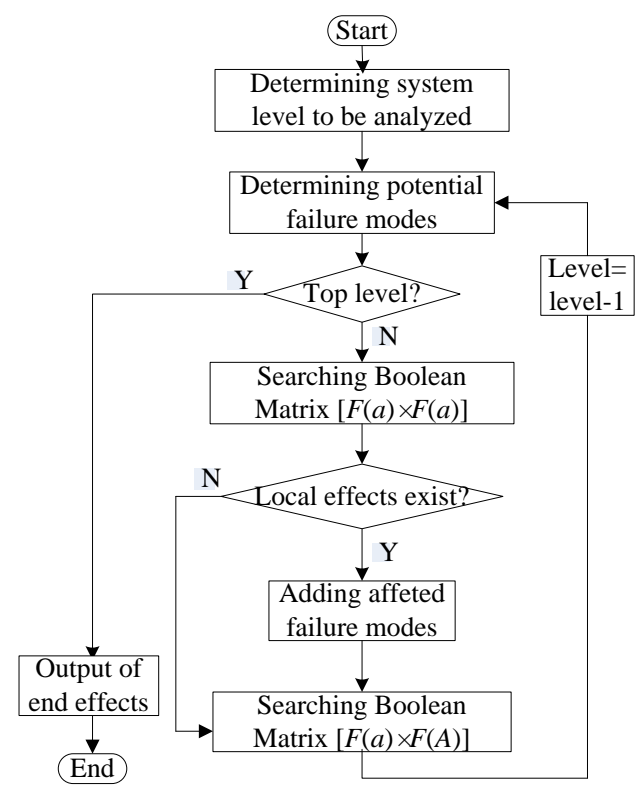

Fig.3 Risk analysis process

Step 1: Determining the system level to be analyzed. Firstly, we can begin the failure effects analysis with the locating the system level. The choosing of the system level is rested with the actual scene and the experience of the analyzer (usually a device or equipment). It is worthless to select the level too lower because it may waste too much energy and time to analyze the unavailable failure modes;

Step 2: Determining the potential failure modes. At this stage, we should find all the possible failure modes, and select the predominant and important failure modes to analyze preferentially. This step is completed with the traditional FMEA, followed by comparison and ranking the FMEA outcome. If the current level is not the top level, then go to the next step, otherwise go to step 5 directly

Step 3: Analyze the local effects. If the interactive influences exist in the same level, we can obtain the local effects by searching the self-correlative reasoning matrix $[F(a) \times F(a)]$. Then, the new failure modes should be added into the candidate failure modes set which need to be further analyzed, otherwise go to step 4 directly; 
Step 4: Analyze the failure effects on the next higher level. Through the step 3, we gain all the possible failure modes that need to be analyzed. We can capture the failure effects of these failure modes on the next higher level by searching the reasoning matrix $[F(a) \times F(A)]$.

Step 5: Export the outcome. Through the cyclic searching operated on the reasoning matrices, the final effects on the whole system can be obtained automatically.

Through the risk analysis process, we can get causal relationship among the failure modes of the components in different level, which is useful for coming into being a FMEA report. As the analysis procedure is reduplicated, and it is easy to be computerized. Of course, we can carry out the fault diagnose with a reverse search direction operating on the Boolean matrices.

\section{Conclusion}

This paper mainly provides a new approach of the formal realization of FMEA knowledge modeling and analyzing for complex electric systems. In our proposed method, the formal knowledge representation model is constructed in which the Boolean matrices are utilized to store the causal knowledge. The new modeling technique could make significant progress in improving knowledge representation and reasoning power for identifying the failure modes and effects for complex electric system. The automated failure effects analysis could be finished with the upward search process along with the Boolean matrices until the initial indenture level arrived. Moreover, the model can also be used to trace original causes of the system damage with the downward consideration.

However, there is a weakness of our proposed method that it is limited to realize the quantitative analysis. Thus, supporting the quantitative analysis requirement through developing our model need be studying in the future.

\section{References}

[1] T.X. Zheng, E. Litvinov: Power and Energy Society General Meeting (Detroit, MI, USA, December 15, 2011). Vol.19, p.1.

[2] R.K. Sharma, D. Kumar and P. Kumar: The International Journal of Quality \& Reliability Management, Vol.22(2005) No.8-9, p.986.

[3] C.J. Price, N.S. Taylor: Reliability engineering and system safety, Vol.76( 2002) No.1, p.1.

[4] R.K. Sharma, D. Kumar, and P. Kumar: The International Journal of Quality \& Reliability Management, Vol.22(2005),p.986.

[5] P.G. Hawkins and D.J. Woollons: Artificial Intelligence in Engineering, Vol. 12(1998), p.375.

[6] P.J. Graham-Jones, B.G. Mellor: Engineering Failure Analysis, Vol. 2(1995) No. 2, p.137. 